\title{
THEATER-WIDE REFERENCE INFORMATION MANAGEMENT
}

\author{
Sandra Berning, Peter Howe, and Todd Jenkins \\ Wright Laboratory \\ Wright Patterson Air Force Base, $\mathrm{OH}$
}

\begin{abstract}
Future military operational concepts emphasize the use of multi-platform operations and the sharing of resources within the theater of operations. This capability would allow many more users access to data from expensive resources and could help extend the operational life of certain aging airframes. However, before concepts for sharing such resources reach their full operational potential, many technical issues must be resolved. Of particular interest and technical challenge are issues related to the processing and sharing of reference systems information. Reference systems information may include position, velocity, attitude, and pointing information from, to, and regarding the ownship, other friendlies, enemy operations, and targets as well as the quality of those parameters. Technical issues that must be addressed include: identification of all sources and users of data including the precision and resolution of that data; requirements for information about the data, such as time tags, and measures of merit; determination of the appropriate levels of data fusion; and a consistent and accurate approach to mutual registration of data from multiple sources.
\end{abstract}

The Reference Systems Branch of the Wright Laboratory Avionics Directorate has conceived a family of programs to address technical issues associated with the fusion and sharing of reference system information in the theater-wide environment. These programs, collectively referred to as TRIM, or Theater-wide Reference Information Management, assume the perspective of a combat aircraft in an operational theater that includes other airborne platforms, overhead assets, and ground based resources. The programs include the Information Management for Theater-Wide Reference Systems, or IMTRS effort, recently completed by The Analytic Sciences Corporation, the Common Reference Frame (CORF) Small Business Innovation Research effort, and a TRIM Program Research and Development Announcement, the latter two to be awarded during FY96. A future advanced development effort, Theater-wide Reference Information Management

Demonstration, will follow these programs and is scheduled to begin in 1998 . This paper provides background information on the current status of airborne reference systems technology and the motivation for extending reference systems concerns to the multi-platform arena. It also highlights several previous technical efforts that form a technical foundation for the TRIM programs and describes current TRIM plans and technical quandaries.

\section{BACKGROUND:}

\section{OVERVIEW:}

Military information fusion technology requirements are currently driven by increasing demands for earlier detection/identification of threats, detection/ discrimination of more complex threats (variety, proliferation, low observability), constantly dwindling financial resources for new military technologies, and increasing sensitivity to own force and neutral collateral damage. These changes have resulted in a renewed emphasis on taking full advantage of all possible data produced by theater and national assets during a military operation. The Wright Laboratory Avionics Directorate has responded to this emphasis with an organizationwide focus on the technologies required to share and fuse information across a theater of operations. The Theater-wide Reference Information Management family of projects was conceived to investigate the various improvements in reference system technology required to support this vision.

\section{REFERENCE SYSTEMS:}

Reference systems determine and extrapolate the state vector for both a vehicle as a whole and for selected critical locations on that vehicle. These state vectors usually include position, velocity, attitude, and time as well as their higher order 
The reference system is also required to perform translations of the state vectors into the most useful coordinate system for each given user of that information. This information is used for functions such as navigation, flight control, sensor stabilization, motion compensation, and pointing, as well as in the fusion of information from related sensors. Current techniques for performing these functions have notable limitations.

As data accuracy requirements become more stringent, airframe structures are becoming more flexible and static misalignments are compounded by misalignments occurring in flight due to aircraft flexure, fuel distribution, and weapon loading. The determination of reference systems information at remote locations on a structurally flexing airborne vehicle is a significant challenge. As an illustration of the complexity of this challenge, one current USAF combat platform has a total of twelve Inertial Measurement Units (IMUs) of various quality mounted around the airframe to generate the state vector information required by the mission sensors and vehicle management systems..

Intensive investments have been made by Wright Laboratory to address various aspects of this problem. These investments have been oriented toward the paradigm of a single aircraft carrying mission sensors and weapons that require state vector information of varying accuracies and data rates to achieve mission requirements. These programs include the Dynamically Sensed Mission Sensor Alignment program, the Integrated Inertial Networks and Vital Advanced Inertial Networks efforts and two Geometric Vector Small Business Innovation Research contracts. These programs have demonstrated the need for far more precise reference data (in particular, velocity, attitude, and time), than is currently available to satisfy mission requirements. Data must be accurate and timely on a single maneuvering airframe at separate mission sensor and store locations such as the aircraft center of gravity, the forward fuselage, and the weapons bays and pylons. The multi-platform environment expands this problem to $\mathrm{n}$-dimensions when the state vector must be valid not only at multiple locations on a single airframe but at multiple locations on multiple platforms within the theater of operations.

\section{DYNAMICALLY SENSED MISSION SENSOR ALIGNMENT}

The Dynamically Sensed Mission Sensor Alignment (DYSMAL) effort explored the impact of dynamic aircraft flexure on the performance of mission sensors onboard a combat aircraft. [1] Although weapon delivery/targeting accuracy is the primary category reliant upon mission sensor alignment, other aircraft subsystems can benefit from an increased level of harmonization and mission sensor "situational awareness", including tactical sensor fusion algorithms which cross correlate information from diverse sensors in an attempt to apply multiple data characteristics to an individual target and multi-ship cooperative missions in which accurate navigation, multiple redundant sensor information, precise directional data, and data interchange between aircraft combine to provide weapon system performance superior to that available from any of the individual aircraft platforms. The primary contribution DYSMAL made to aircraft system designers is an increased awareness of the interdependencies between structure, avionics sensor behavior, and weapon system performance. DYSMAL results showed that designers of the newer more flexible airframes, and of retrofit programs which place high accuracy sensors onboard older platforms must acknowledge the increased effects of static and dynamic airframe bending upon the avionics and weapon delivery systems, or face the consequences of degraded weapon system performance.

\section{INTEGRATED INERTIAL NETWORKS}

The objective of the Integrated Inertial Network (IINET) program was to design a configuration of inertial and/or direct flex sensors of varying precision, taking into account dynamic airframe flexure and system redundancy, to produce accurate, dynamic corrections for aircraft subsystems distributed throughout the airframe as well as producing a more accurate, fault tolerant, integrated navigation solution.

The program determined specifications for the inertial sensors required at each location on the airframe and resulted in a detailed set of system design metrics for performing system trade-offs. Analysis performed during this effort revealed dynamic alignment accuracy requirements at selected locations on the airframe as stringent as .25 milliradians and update rates as frequent as $200 \mathrm{~Hz}$. These were used to select a preferred configuration for a fighter attack aircraft. The recommended IINET configuration minimizes overall system cost while providing reliable, fault tolerant performance sufficient to support advanced mission requirements at distributed locations on a single airframe. In addition, the need for further technical developments was identified, in particular in 
the area of techniques for dynamically compensating for aircraft flexure and vibration. Reference 2 contains more details on inertial network architectures, system requirements, and flight test results.

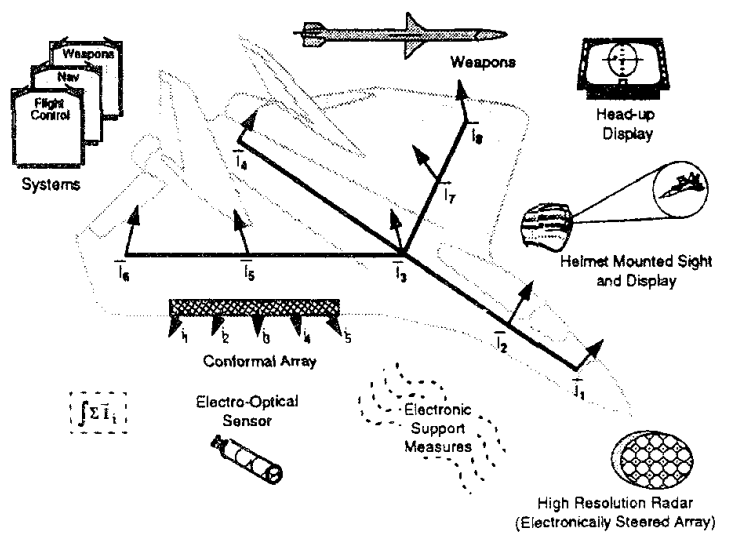

Figure 1: Integrated Inertial Networks

\section{VTTAL ADVANCED INERTIAL NETWORK}

The Vital Advanced Inertial Network (VAIN) program is an ongoing effort to develop an Advanced Inertial Network, taking advantage of multiple inertial sensors, flex sensors, and the Global Positioning System (GPS) to provide valid data at distributed airframe locations to support precision targeting and weapon delivery requirements. The design metrics and requirements established under the IINET program form the technical foundation for this advanced development effort.

VAIN comprises sensors and algorithms that harmonize flight control, navigation, targeting, attack, and weapons for precision strike, survivable air-to-air engagements, and other precise multi-sensor applications. VAIN also provides fault tolerant inertial reference for flight control and navigation, dynamic alignment for targeting and attack, and weapon initialization. Particular emphasis is being placed on developing technology that dynamically compensates for in-flight aircraft flexure and vibration.

\section{FUSION OF AVIONIC SENSOR INFORMATION INTO A GEOMETRIC VECTOR}

The objectives of the Phase I Small Business Innovative Research contract entitled, "Fusion of Avionics Sensor Information into a Geometric Vector" with GPS [3] were to improve mission sensor performance, navigation information, and overall weapon system performance through deep integration. The Geometric Vector used in this study consists of position, velocity, attitude, and time. The term deep integration is defined as the inclusion of states for several sensors within a tightly-coupled centralized extended Kalman filter. Several reconnaissance and strike missions were investigated in which the deep integration concept could be exploited. A candidate sensor, the Synthetic Aperture Radar mode, was selected for inclusion in an extended Kalman filter with GPS and INS systems. Error models developed in previous Wright Laboratory research were modified to include the Quaternary Phase Error (QPE) from the SAR. Study results revealed that this approach achieved enhanced navigation performance for the weapon system. For example, GPS/INS/SAR integration was much more resistant to GPS jamming and outages. Using SAR range, range-rate, and $\mathrm{QPE}$ measurements, the navigation solution remained bounded for significant intervals even in the advent of complete GPS outages.
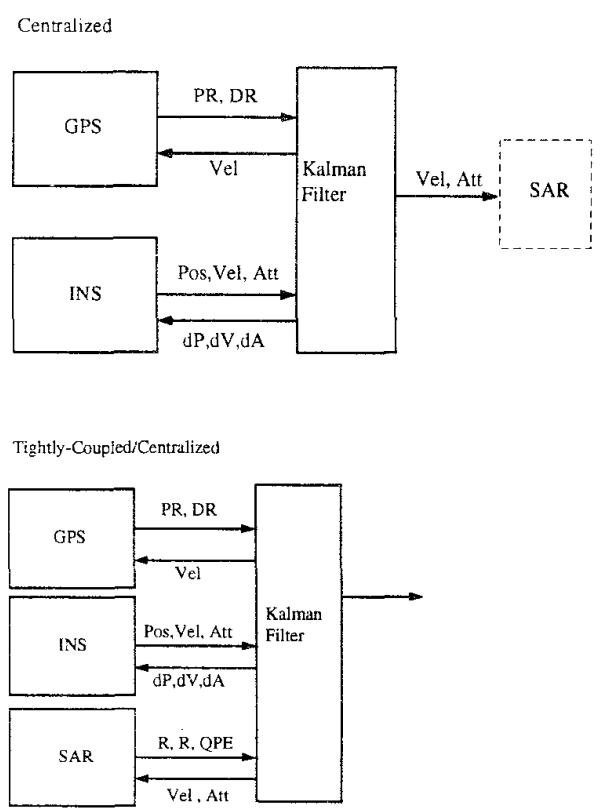

Figure 2: Levels of GPS/INS/SAR Integration

Another significant benefit resulting from the GPS/INS/SAR integration was the increase in SAR mapping resolution. The velocity accuracy from this system was more than a magnitude better than that of an INS alone. This resulted in a proportionate 
increase in SAR map accuracy. Phase II will involve simulation and design work to produce an EGISAR (Embedded GPS/INS/SAR). An existing Embedded GPS INS (EGI) unit, currently being procured for 14 DOD platforms, will be used.

\section{REFERENCE SYSTEMS IMPACT ON THEATER-WIDE SENSOR FUSION}

The recent history of precision reference systems has been to create continually higher levels of physical, functional, and informational integration within the navigation/reference system, between that system and other onboard systems, and with the crew. The most stringent performance demands on this integration have been generated by the requirements of onboard mission sensors such as SAR or ElectroOptic (EO), and the desire to harmonize and fuse their data [4, 5]. The logical continuation of this trend is to support information integration across multiple platforms (airborne, orbital, ground based, and sea based) separated in both time and space. This level of integration will enable far reaching sensor and information fusion for battlefield management, resource and environment monitoring, and geophysical monitoring. Reference system behavior and performance can impact the effectiveness of three aspects of the fusion process: the performance of individual sensors, the registration problem, and uncertainty about uncertainty.

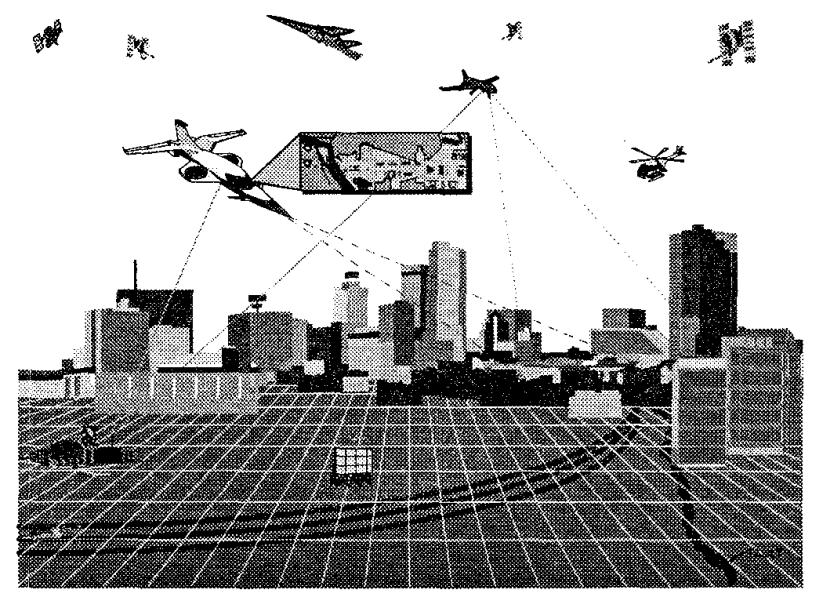

Figure 3: Geophysical/Environment Monitoring Enabled by Reference Systems

The performance of the individual sensors being used in fusion can be severely influenced by the reference system supporting those sensors. The performance of SAR is a classic example, with both focus and contrast being dependent on high frequency reference information, and azimuth error being a function of absolute velocity error $(1 \mathrm{~cm} / \mathrm{sec}$ velocity error is approximately 0.1 milliradian of azimuth error at 400 knots). Analysis and flight tests conducted in the Wright Laboratory GPS Exploitation program $[6,7,8]$ and its follow-on efforts have verified this latter effect and also revealed that dramatic reductions are possible in SAR height above target errors by the judicious use of improved velocity reference, internal radar measurements, and trajectory shaping. This latter point has special relevance for some fusion problems and will be discussed later.

Obviously, position errors of individual sensors affect the fusion registration problem. Less obvious is the effect that other elements of the reference state vector can have. Sensors that provide measurements in the form of angular bearings are influenced by attitude errors in the reference vector at the sensor location. The SAR azimuth error caused by vehicle velocity error has the same impact. Sensors that use Doppler measurements are also subject to velocity error effects. Any attempt to quantify the errors induced by the individual platform state vectors quickly becomes entwined in the first and second order error equations of the sensors being used.

The effects of uncertainty in reference system state vector information are also difficult to deal with quantitatively. Modern military integrated reference systems have eigenvectors of uncertainty that behave in a complex fashion as a function of vehicle dynamics, external environment (such as jamming), and time. The eigenvector is then coupled through the error processes of the sensor (e.g.. SAR, EO) measurement to produce the ultimate true uncertainty of the sensor data. This process was exploited in the WL Northrop study [8] of precision SAR targeting to generate trajectory shaping and measurement scheduling that resulted in orthogonal uncertainty vectors producing the best possible target location. Historically, integrated reference system algorithms have been tuned to minimize the errors in the state vector, with quantitative knowledge of the errors being a by-product of this process. It is not clear what quantitative improvement would result in sensor fusion if this information was more accurately computed. 


\section{THEATER-WIDE REFERENCE INFOR- MATION MANAGEMENT (TRIM) PROGRAMS}

\section{OVERVIEW}

The TRIM family of programs will address the aforementioned reference system issues and will identify others needing to be addressed in order to satisfy technical requirements for multiple platform operations. It will also develop analytical techniques and necessary reference systems technologies, and will demonstrate these technologies through a series of increasingly complex simulations. The goals of these technology development efforts will be to enable maximum use of both on- and off-board resources for reconnaissance, targeting, weapon delivery, and situational awareness. Technical investigations will address processing and sharing of reference systems information (position, velocity, attitude, flexure, and time) related to ownship, other friendlies, terrain, threats, enemy operations, and targets collected using radar, electro-optic, and electronic intelligence (ELINT).

This section contains a summary of the goals and technical approach for each member of this family and a preliminary discussion of the technical accomplishments of the IMTRS program.

\section{INFORMATION MANAGEMENT FOR THEATER-WIDE REFERENCE SYSTEMS (IMTRS)}

The objectives of the IMTRS effort were to establish the limitations and quantitative sensitivities associated with real-time algorithms and data structures currently available for theater-wide reference information management, and to establish which classes of these algorithms and data structure implementations require technical development. The intent was to identify requirements for future technical developments in the areas of reference systems technology and analytical techniques that would enable maximum use of both on-and off-board resources. Pertinent resources include information regarding the ownship, other friendlies, terrain, threats, enemy operations, and targets collected using radar, electro-optic, and electronic intelligence (ELINT). It was assumed that the targeting combat aircraft would be the central point for all data processing and fusion.
The program produced a fusion performance model which was exercised with multiple parameter sets. Performance predictions for each set helped determine overall performance sensitivity. The fusion algorithm requirements were defined by investigating theater-wide fusion algorithms with respect to platform reference system performance. This analysis helped identify the data structure that must be available to allow the targeting platform to execute its fusion algorithms. During this analysis, data structure requirements for image, feature, and target level fusion were also considered. Next, key performance for both reference and sensor systems deployed on the platforms specified by the baseline scenario were investigated. Reference system capabilities quantified included platform state (position, velocity, estimation, acceleration, and platform inertial) estimation accuracies, sensor pointing estimation accuracy, and motion compensation effectiveness. Mission operational characteristics such as platform altitude and speed for the scenario were also characterized. Other parameters characterized included ground resolution (pixel size on the ground), angle-of-arrival estimation accuracy, sensor field-of-view (image size), target detection range, sensor temporal sampling rate, sensor pointing accuracy, and jitter. A capability was developed to quantify reference system parameter sensitivities of the target state estimation accuracy achievable based on the fusion algorithms which utilize the target state measurements provided by the sensor platforms.

Baseline scenarios were developed which included trajectories for each of the platforms, scenario geometry, time, and all other relevant scenario characteristics including target range, elevation and azimuth data plus sensor viewing parameters. A model was then developed to predict the performance of various fusion algorithms as a function of multiple parameters, especially reference systems quality. The primary performance metric provided by the model was target location uncertainty for the fusing platform. Overall system performance could be determined based on the target location uncertainty and the munition accuracy. Target location uncertainty was derived from the covariance of the fused target location estimate and the changes in covariance with time and with respect to each platform target report. 


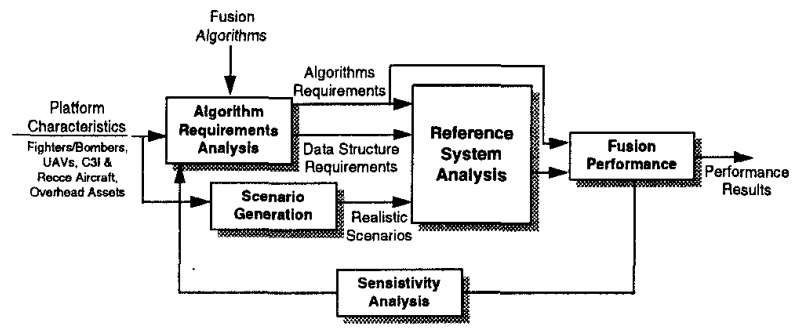

Figure 4: IMTRS Analysis Approach

A sensitivity analysis was performed which helped determine the sensitivity of the performance model to platform reference system performance, fusion algorithm performance, ownship reference system performance, sensor system performance, and scenario geometry. Platform and ownship reference system performance sensitivities were quantified by varying the covariance of the platform location. Likewise, sensor system performance was explored by analyzing the sensitivity to individual platform sensor system performance. Also, the effect of fusion algorithm performance was analyzed by varying the overall platform measurement covariance to indicate the ability of fusion algorithms to extract all available information from platform sensors. Finally, a representative set of scenarios, extracted from the baseline scenario showed the sensitivity of the algorithm performance to platform viewing geometry.

\section{COMMON REFERENCE FRAME FOR MULTI- PLATFORM OPERATIONS (CORF)}

The objective of the CORF effort is to develop technology that will support the sharing and fusing of reference systems information based upon multiple reference frames and geographic datums while preserving the inherent accuracies with respect to the unique models of the surface of the earth that exist within those datums [9]. The goal is a consistent and accurate approach to mutual registration of data from multiple sources. This effort will examine existing and projected sources and users of reference systems data, the accuracy and other pertinent attributes of that data, and the reference frames and/or geographic datums employed. In order to identify the information content of the data to be shared across platforms, it will also be necessary to determine the required parameters and the levels of data fusion to be performed by each data source, as well as the required resolution and measures of merit associated with all data to be shared. Through the use of simulation, a demonstration will be conducted of common reference frame technology during a representative battlefield scenario. Resulting technology will form the basis for effective fusion of reference system information from multiple sources, across a cooperating set of users.

\section{TRIM CONCEPT DEVELOPMENT}

This effort will develop the reference systems technologies needed to support the theater-wide sharing of reference systems resources and will evaluate those technologies using analysis and a series of increasingly complex nonreal-time simulations. Mission scenarios will be identified for which multi-platform operations could play a key role, such as deep interdiction or deep counterair. Selection of information sources and users will be based upon those scenarios and could include various manned and unmanned airborne platforms, overhead assets, deployed ground sensors, and associated weapons. Technical issues to be addressed include determination of: (1) sources and users of reference systems data in the theater of operations including the precision and resolution of that data; (2) requirements for information about the data, such as time tags and measures of merit; (3) the levels of data fusion to be performed by each source and each user of the data and the reference systems parameters required to support the fusion algorithms; and (4) a consistent and accurate approach to mutual registration of data from multiple sources, taking into account the existing reference frame(s) and geographic datums being used by both the sources and the users of the data.



Figure 5: TRIM Air-to-Ground Scenario

\section{FUTURE DIRECTIONS}

Work performed by this organization to date has provided indications of technical areas that require 


\section{FUTURE DIRECTIONS}

Work performed by this organization to date has provided indications of technical areas that require further exploration. It is expected that these indications will evolve and become more focused as a result of the technical explorations of the TRIM programs as well as concurrent sensor fusion research taking place within DOD, industry, and academic laboratories. Three areas of future interest are briefly discussed below.

First, there is an urgent requirement for better metrics. While previous work has provided a good understanding of the quantitative sensitivity of individual mission sensors to reference system errors, the question of quantitative sensitivity of fusion processes to these errors is only now beginning to be addressed. Obviously, an ability to decide which improvements to seek, and how effectively an improvement has been achieved is largely dependent upon this point. Just as obviously, it is not possible to determine such metrics without the collaboration and collective insight of the larger sensor fusion community.

Secondly, there are numerous well defined coordinate and geodetic reference frames with associated datums (a handheld GPS receiver recently purchase by one of the authors incorporates 103 different datums in its data base!). In the past, the problem has been one of gross discrepancies due to inadvertently mixing datums. The problem of the future becomes one of selecting the most appropriate coordinate frame/datum for each stage of the fusion process and of ascertaining the numerical accuracies that need to be maintained during transformations. This is not as straight forward as one would hope, with recent experience on operational systems highlighting numerical instabilities and error build up in long duration missions. It is expected that this area will be revisited repeatedly as the sophistication and accuracy requirements of fusion efforts evolve.

Finally, the concept of accurately assessing uncertainty and exploiting it as part of the measurement process and sensor management process is embryonic. Further development of this concept requires a more detailed understanding of the dynamics and mathematics relating the mission sensor's error uncertainty with the contribution due to the reference systems involved, and the subsequent effect of the fusion algorithms in use. Future research will be required to ascertain the quantitative benefits of improving knowledge of uncertainty and the most beneficial methods of exploiting this information.

\section{REFERENCES}

[1] Berning S., Abernathy D., "Applications of Dynamically Sensed Mission Sensor Alignment Program (DYSMAL)", Proceedings of 21st Joint Services Data Exchange (JSDE) for Guidance, Navigation and Control (GN\&C), Palm Springs, CA October 1992, pp. 474-485.

[2] Kelly R., Carlson N., Berning S., "Integrated Inertial Network", Proceedings of I.E.E.E. Position Location and Navigation Symposium, PLANS-94, Las Vegas, NV April 1994, pp. 439-446

[3] Kirk, J., "Fusion of Avionics Sensor Information into a Geometric Vector with Global Positioning System (GPS), SBIR Phase I Final Report, WL/AA Phase I SBIR, Technology Services Corp., Dec 1995.

[4] Lewantowicz Z., Paschall R., "Deep Integration of GPS, INS, SAR and other Sensor Information", Advisory Group for Aerospace Research \& Development (AGARD) Guidance and Control Panel, AGARD-AG-331, June 1995, pp. 231-264.

[5] Layne J., Paschall R., "Performance Analysis of an Integrated SAR/GPS/INS Targeting and Navigation System", WL/AAAS Technical Memo, October 1993.

[6] Ioannidis G., Walton N., Pujara N., Bublitz C., "GPS Exploitation for Precision Targeting: A Relative Targeting System," Proceedings of I.E.E.E. Position Location and Navigation Symposium, PLANS-94, Las Vegas, NV April 1994, pp. 145-152.

[7] Beck G., et al, "GPS Exploitation for Precision Targeting: Tactical Applications", Proceedings of ION 49th Annual Meeting, Cambridge, MA June 1993, pp.183-192.

[8] Abbott A., Pujara N., Howe P., "GPS Exploitation for Precision Targeting - Observability Using Synthetic Aperture Radar", Proceedings of ION 49th Annual Meeting, Cambridge, MA June 1993, pp.193-202.

[9] "Common Reference Frame for Multi-Platform Operations", Topic AF96-118, Program Solicitation 96.1, FY 96 Small Business Innovation Research (SBIR) Program, pp. AF-112, AF-113. 